\title{
Advances in the Treatment of Neutropenia
}

\author{
David C. Dale, MD \\ Department of Medicine, University of Washington School of Medicine, Seattle, WA
}

\begin{abstract}
Purpose of review-This review updates treatment of neutropenia from articles published from January 2008 through April 2009.

Recent findings-Chemotherapy-induced neutropenia occurs most commonly in the first cycle of treatment. Older patients, patients with multiple co-morbidities, and those receiving more myelotoxic drugs are prone to develop neutropenia and its complications. Current guidelines recommend use of the myeloid growth factors for the first cycle of chemotherapy for patients with more that a $20 \%$ risk of febrile neutropenia. Meta-analysis from randomized trials shows that granulocyte colony-stimulating factor (G-CSF) prophylaxis is associated with patients receiving more intensive chemotherapy, having better survival, but also having a higher risk of secondary AML. Antibiotic remain the mainstay of treatment of febrile neutropenia and are increasingly used for prophylaxis in "low risk" patients. Diagnosis and treatment of other type of neutropenia is also steadily improving.
\end{abstract}

Summary-The myeloid growth factor G-CSF has radically changed our approach to the management of neutropenia. Antibiotics remain the mainstay of treatment of febrile neutropenia.

\section{Keywords}

neutrophil; neutropenia; granulocyte colony-stimulating factor (G-CSF); chemotherapy-induced neutropenia

\section{Introduction}

Neutropenia, an absolute neutrophil count (ANC) less than $1.5 \times 10^{9}$ per liter, occurs in many clinical settings. For many years, management of neutropenia was limited to the prompt prescription of antibiotics when patients with neutropenia, usually those with an ANC $<0.5 \times 10$ 9/L, developed a febrile illness. The development of the hematopoietic growth factors, particularly granulocyte colony stimulating factor (G-CSF), greatly altered our approach to the problem of neutropenia. This review focuses on advances published January 2008 through April 2009.

\section{Chemotherapy-Induced Neutropenia}

Neutropenia remains the principal dose-limiting toxicity for cancer chemotherapy.

Neutropenic complications include fever, superficial and deep tissue infections, bacteremia, sepsis and death. Because of concern about these complications, the intensity of chemotherapy is often reduced, increasing the likelihood that the cancer treatment will not be fully effective. Recently, careful studies have defined the risk factors for chemotherapyinduced neutropenia, based on systematic review of the literature, chart reviews and

Address correspondence to: David C. Dale, M.D., Professor of Medicine, Department of Medicine, University of Washington, Box 356422, 1959 NE Pacific St., Rm AA522, Seattle WA 98195, Tel 206-543-7215, Fax 206-685-4458, dcdale@u.washington.edu. 
prospective studies. Risk factors can be classified as: patient-specific factors, e.g., age and co-morbidities, disease-related factors-type of cancer and the expected myelotoxicity of the planned chemotherapy. Definition of risk factors is very important to plan and administer appropriate chemotherapy, to decide on the supportive care measures needed, and to maximize the benefits of treatment while minimizing its costs.

A prospective study of patients with breast, lung, colorectal, ovarian cancer or lymphoma treated in community setting provided a new database for analyzing the risk of neutropenic complications with cancer chemotherapy. (1) The study collected data from baseline and through four cycles of treatment. Thirty-seven percent of the patients were 65 years old or greater and $27 \%$ had one or more comorbidities. Approximately $24 \%$ of patients had reductions in the intensity of treatment to less that $85 \%$ of the standard dose in the first cycle of treatment. Despite these dose reductions, neutropenia and febrile neutropenia were common, and approximately $60 \%$ of all episodes of febrile neutropenia occurred during the first cycle of treatment across tumor types, disease stage and chemotherapy regimens. (1) As yet there is no clear pathophysiological explanation for the greater risk of febrile neutropenia in the first cycle. It may be due to the common practice of reducing the intensity of chemotherapy in subsequent cycles when patients develop neutropenia in the first cycle, changes in proliferative activity or numbers of myeloid progenitors after exposure to the first cycle of treatment or to changes in the pharmacokinetics of the chemotherapy drugs.

Based on data from this prospective study, Lyman, et al analyzed the risk of neutropenic events for 3760 patients starting a new chemotherapy regimen at 115 randomly selected community oncology practices across the U.S. (2) The authors used a split sample approach to carefully determining specific risk factors associated with first cycle severe or febrile neutropenia and create a model for predicting the risk of chemotherapy-induced neutropenia. This analysis showed that older age, prior chemotherapy, abnormal hepatic or renal function, low pre-treatment white blood cell count, concomitant immunosuppressive medications and planned relative dose intensity greater than $85 \%$ of standard dosing are key risk factors. In this analysis, prophylactic G-CSF significantly reduced the risk of severe and febrile neutropenia. Other studies show similar results. $(3,4)$

\section{Neutropenia in Children with Cancer}

A Swiss single-center, cohort study reported on 360 pediatric cancer patients with 424 years of cumulative chemotherapy exposure. (5) The patients experienced 629 episodes of febrile neutropenia (approximately 1.5 episodes per year) and 145 episodes ( $23 \%$ ) of bacteremia.

Risk factors for febrile neutropenia included early stage/first cycle of treatment, dose-intense chemotherapy, marrow involvement, central venous catheters and prior episodes of febrile neutropenia. A preliminary risk model was developed from this study. Another study showed similar results. (6)

\section{Infections Associated with Chemotherapy-Induced Neutropenia}

Invasive fungal infections are serious consequences of protracted severe neutropenia, particularly during treatment of acute myeloid leukemia (AML) or after hematopoietic stem cell transplantation. A study from Barcelona, Spain pointed to the changing pattern of invasive fungal infections. (7) The risk for aspergillosis is greatest for patients receiving corticosteroids or other immunosuppressive agents, with graft vs. host disease or with persistent neutropenia after transplantation with cord blood cells, mismatched allogeneic or T-cell depleted progenitor cells.

The risk of invasive aspergillosis has also recently been analyzed in a retrospective review of 385 patients treated at the University Hospital of Strausbourg, France. (8) Twelve week 
survival was only $52 \%$. Risk factors for severe infections were: allogeneic transplantation, progression of the underlying malignancy, pre-existing respiratory disease, treatment with corticosteroids, renal impairment, and low monocyte counts. Neutropenia was associated with a much higher attributable mortality.

A third recent report analyzed treatment strategies for care of patients at high risk of invasive fungal infections, e.g., prophylaxis, empiric or targeted treatment. (9) The authors recommended prophylactic treatment with posaconazole in patients undergoing allogeneic transplantation and after induction chemotherapy for AML, and they recommended empiric therapy for persistent febrile neutropenia with caspofungin or liposomal amphotericin B. Voriconazole is their preferred treatment for proven aspergillosis. The report also emphasizes the need for better diagnostic strategies for early detection of invasive fungal infections.

In addition to the expanding array of fungal infections, infections by both Staphylococcus areus and gram-negative bacilli remain critical problems. (10) A recent Korean study described E. coli bacteremia in 508 patients and Pseudomonas aeruginosa bacteremia in 106 cases. Severe neutropenia, indwelling central venous catheters, and presentation with frank septic shock were predictors of Pseudomonas infection. The thirty-day mortality rates were $26 \%$ and $13.6 \%$ for Pseudomonas and E. coli bacteremia, respectively. This analysis also pointed to the importance of prompt and appropriate antibiotic treatment, including empiric coverage for Pseudomonas, for patients with signs of sepsis associated neutropenia.

\section{Patients at Low Risk of Neutropenic Complications}

Many patients undergoing cancer chemotherapy are deemed to be at low risk for febrile neutropenia. For these individuals, oral, out-patient antibiotic treatment is now deemed appropriate. A review reported no difference in the clinical failure rates or mortality for ambulatory regimens compared to standard hospital-based treatments in low risk patients. (11) An absolute neutropenia count $<0.1 \times 10^{9}$ per liter was predictive of failure of failure of outpatient therapy. (11) Other key determinants of the risk of outpatient treatment are: the hemodynamic status, co-morbidities, and signs of organ failures. Also patients with nausea and vomiting often cannot be reliably treated with oral agents.

A survey of practice patterns showed that oral antibiotics and out-patient therapy are now a common practice for low risk patients with febrile neutropenia. (12) A survey of 1207 oncologists showed that $82 \%$ now use oral antibiotics for low risk patients. The respondents indicated that they selected patients for out-patient therapy who are younger, had higher blood neutrophils, no infiltrate on a chest $x$-ray and lived closer to health care provider. The commonly used drugs were levofloxacin (20\%), ciprofloxacin (36\%), and ciprofloxacin plus amoxicillin/clavulanie (35\%).

A study from the loannina University Hospital in Greece reviewed care for 1372 older patients at risk of chemotherapy-induced neutropenia, utilizing the database of the Hellenic Cooperative Oncology Group. (13) This study focused on relatively healthy elderly adults (median age of 70 years) diagnosed with breast, colorectal or lung cancer. Three year median survival rates were $41 \%, 25 \%$ and $31 \%$, respectively. Overall, the study showed that otherwise healthy, older patients with advanced cancer can safely tolerate modern chemotherapy.

\section{Chemotherapy Dose Intensity, Neutropenia and Patient Outcomes}

Neutropenia is also important in cancer treatment because concern about this complication drives clinicians to reduce the chemotherapy dose intensity. In a careful review, Lyman 
emphasized the importance of standard or full dose chemotherapy for responsive malignancies, particularly for lymphoma and breast cancer. (14) For these malignancies, there is a reduction in long-term survival in these patients receiving less than $85 \%$ of the standard treatment. Kahn et al also reported on dose reductions in chemotherapy as a consequence of neutropenia. (15) Several recent studies referenced in these papers show an association of myelosuppression during chemotherapy and improved overall survival, the inference being that myelosuppression is a surrogate for sufficient intensity of therapy for it to be effective.

\section{Neutropenia and Quality of Life}

The onset of severe neutropenia is usually soon followed by fever, myalgias and flu-like symptoms. Hospitalization for febrile neutropenia or sepsis is a major setback for patients undergoing cancer chemotherapy. For several years, Cella et al have worked on developing standardized methods for measuring neutropenia-specific quality of life measures that can be applied in studies of cancer chemotherapy. A recent report summarizes development of the FACT-N or the Functional Assessment of Cancer Therapy-Neutropenia. (16) This 19 item test assesses symptoms associated with neutropenia and should be a useful instrument for studies of chemotherapy-induced neutropenia.

\section{Myeloid Growth Factors for Treatment and Prevention of Chemotherapy- Induced Neutropenia}

The myeloid growth factors have had a major impact on the management of neutropenia. During the past year updated recommendations were made by expert panels in Canada and Europe. $(17,18)$ These recommendations closely parallel those from the American Society of Clinical Oncology (ASCO) and the National Comprehensive Cancer Network (NCCN) and a recent Cochrane analysis. (19-21) The important role of oncology nurses in the development and implementation of these guidelines was also emphasized in a recent report. (22) Consistently these guidelines point to the benefits of G-CSF for prevention of febrile neutropenia and the appropriateness of its use in patients with co-morbidities having a risk of severe and febrile neutropenia greater than about 20 percent.

Several recent papers addressed the cost effectiveness of primary vs. secondary prophylaxis with myeloid growth factors. Most recent studies have focused on pegylated G-CSF or pegfilgrastim. A study by Ramsey, et al estimated that the incremental cost effectiveness ratio (ICER) of pegfilgrastim as primary vs. secondary prophylaxis for women with earlystage breast cancer undergoing chemotherapy was $\$ 48,000$ per episode of febrile neutropenia avoided. (23) For this analysis, the clinical data and assumptions came from previous randomized, controlled trials. The factors identified as the most influential in the model were the predicted case fatality, the relative risk reduction with primary prophylaxis and the age of the patient. Another analysis by Eldar-Lissai, et al indicated that prophylactic pegfilgrastim was not only cost effective, but cost saving in most common clinical and economic settings in the U.S. (24) Timmer-Bonte, et al, however, emphasized that secondary prophylaxis with antibiotics is the least expensive strategy for managing the risk of febrile neutropenia and that antibiotics plus G-CSF are a much more expensive strategy based on patterns of care and costs in the Netherlands. (25) To a large degree, the differences in these analyses relate to the estimated cost of hospitalization in different locales and secondarily to the estimated risk of febrile neutropenia in the population at risk.

The economics of therapeutic use of G-CSF was recently reviewed by Cosler, et al, building on the results of a recent meta-analysis of randomized control trials of the use of G-CSF of treatment of febrile neutropenia. (26) Using U.S. healthcare data, the study showed that 
there was a net savings with the therapeutic use of G-CSF, principally due to shortening the duration of hospital stays. This strategic advantage of G-CSF use needs further evaluation.

\section{Secondary Leukemias and Chemotherapy-Induced Neutropenia}

Lyman, et al reported on the risk of AML and MDS in patients receiving chemotherapy with or without G-CSF in a meta-analysis based on data FROM randomized control trials. (27) Based on published data for randomized clinical trials involving a total of more than 12,000 patients with a median follow-up of 54 months, secondary malignancies were reported in $3.3 \%$ and $3.2 \%$ with and without G-CSF treatment, respectively. The relative risk of AML/ MDS was higher for those receiving G-CSF, however, there was a survival advantage for this group as well, probably attributable to the patients receiving overall more consistent and intensive chemotherapy for their malignancies.

\section{Clinical Guidelines and Clinical Priactice}

It is probably not surprising that audits of clinical practice show that there is considerable variation in the cancer treatments and many ways in which practice can be improved. A retrospective chart review of a random sample of 305 cancer patients in one large U.S. center showed that practices were often not aligned with established practice guidelines related to the prevention and management of the risk of neutropenia. (28) A Brazilian study showed that development of critical pathways for the management of chemotherapy-related febrile neutropenia served to improve outcomes. (29)

\section{Neutropenia Induced by Non-Chemotherapy Drugs}

A major literature review of non-chemotherapy drug-induced neutropenia found 980 reports of agranulocytosis or severe neutropenia associated with 125 drugs. (30) The incidence is about 10 cases per million per year. (31) The most frequently associated drugs were: carbimazole, clozapine, dapasone, dipyrone, methimazole, penicillin G, procainamide, propylthiouracil, rituximab, sulfasalazine and ticlopidine. (30) An ANC less that $0.1 \times 109 /$ $\mathrm{L}$ had a case fatality rate of $10 \%$. The summarized data indicated that patients treated with a myeloid growth factor had shorter duration of neutropenia and fewer infectious or fatal complications.

\section{Neutropenia with Interferon Alpha 2A and 2B}

The interferons are widely used in the treatment of hepatitis, multiple sclerosis and other inflammatory conditions. Antonini et al (32) reported on occurrence of neutropenia in 319 patients receiving peg-interferon 2 alpha and interferon 2 beta with ribavirin for chronic hepatitis C. Neutropenia occurred in $17 \%$, but there were few infectious complications. This study suggests that mild to moderate interferon-induced neutropenia can be tolerated and therapy continued in most cases.

\section{HIV and Neutropenia}

Babadoko et al (33) reported on the frequency and severity of neutropenia in HIV infected patients in Nigeria. In 400 consecutive symptomatic and asymptomatic patients, there was a significant correlation of CD 4 counts and the ANC. Ortega et al reported the patterns of bacteremia in HIV patients. (34) In a study of 1077 patients, $60 \%$ of isolates were cotrimoxazole resistant, probably due to widespread use of this agent for prophylaxis. Significant risk factors for bacteremia were: neutropenia, cirrhosis and corticosteroid therapy, and the mortality rate was $13 \%$. Although the problem of neutropenia in HIV infected patients has decreased in some places, it has not gone away. 


\section{Congenital Neutropenia and Leukemia}

Rosenberg et al updated their studies on the risk of AML in patients with congenital neutropenia on long- term treatment with G-CSF. (35) In the majority, but not all, of these patients neutropenia is attributable to mutations in the gene for neutrophil elastase, the ELA2 gene. This analysis showed that leukemia occurs with about equal frequency in patients with and without ELA2 mutations. (35) There is a growing list of genetic causes for congenital neutropenia $(36,37)$.

\section{Neutrophil/Granulocyte Transfusions}

G-CSF in combination with corticosteriods (usually dexamethasone) will increase the ANC to $20-40 \times 10^{9} / \mathrm{L}$ in about 4 to 6 hours, counts high enough to permit collections of $10 \times 10^{9}$ cells by leukapheresis for transfusion therapy of severe infections in neutropenic patients. Seidel et al reported on a randomized control trial enrolling 74 adult patients in Germany that showed no benefit (or harm) to transfusions with neutrophils from G-CSF stimulated donors. (38) Survival was approximately $80 \%$ in both arms of the study and the duration of neutropenia was relatively short in the control arm. Ofran et al reported from Israel on 47 patients receiving 348 consecutive transfusions for life threatening infections. (39) The transfusions appeared to be safe but efficacy could not be assessed because there was no control group. Taskinen et al reported improved engraftment in 22 patients receiving leukocyte transfusions after stem cell transplantation. (39) There is now an ongoing multicentered, randomized control trial in the U S, sponsored by the NIH. (40)

\section{Granulocyte Macrophage Colony Stimulating Factor and Neutropenia}

Granulocyte-macrophage colony stimulating factor (GM-CSF) shares many in vitro properties with G-CSF in hematopoietic cell culture systems. Studies in patients and normal volunteers, however, demonstrated that these cytokines have major differences in the effects on blood cell formation and deployment and in their potencies to stimulate neutrophil formation. G-CSF is by far the more potent stimulus. $(41,42)$ Almost all of the evidence for for the use of myeloid growth factors for prevention of febrile neutropenia comes from studies of G-CSF. (43) GM-CSF is generally not used for the treatment of most forms of chronic neutropenia because of the lack of efficacy and its association with more severe adverse effects. There were no reports of randomized or controlled trials of GM-CSF for the treatment or prevention of neutropenia during the review period January 2008-May 2009

\section{Biosimilars and Other New Agents}

On the horizon are several new agents for stimulation of neutrophil production, taking advantage of all that has been learned over the last 25 years in the development of G-CSF and GM-CSF. (44-45) The primary focus is on new manufacturers making G-CSF. A current controversy is how to prove that the new products are safe and effective before they are licensed for medical applications.

\section{Additional Drugs of Interest}

Many newer anti-cancer drugs have been developed with more targeted effects than older chemotherapeutic agents. Despite these efforts, many of these agents have myelosuppressive effects, illustrating the complexity and fragility of the normal process of production, maturation and deployment of neutrophils and monocytes. Some examples are: 


\section{Rituximab and neutropenia}

Rituximab is a chimeric monoclonal antibody directed to CD20 and widely used in the treatment of non-Hodgkin lymphoma and as a non-specific immunosuppressive agent. Lateonset grade 3 or 4 neutropenia occurs in about 10- to $15 \%$ of patients with a median time to onset of about 4 months. The neutropenia is mild and treatment with myeloid growth factors is usually not required. (46)

\section{Lenalidomide and neutropenia}

Lenalidomide is an immunomodulatory drug, structurally related to thalidomide, with multiple mechanisms for its anti-angiogenic and anti-neoplastic properties.(47) In clinical trials, grade 3 and 4 neutropenia were observed in $32 \%$ of patients receiving this agent plus dexamethasone. (48)

\section{Bortezomib and neutropenia}

Bortezomib is a member of a novel class of therapeutics agents which reduce NF- $\mathrm{kB}$ mediated IL-6 production. (47) It induce apoptosis of multiple myeloma cells presumably through transcriptional down-regulation of growth/survival signaling pathways and up regulates apoptotic cascades, ubiquitin/proteasome pathways, and heat-shock proteins. In controlled clinical trials, thrombocytopenia and neutropenia are important toxicities, probably through the same mechanisms that suppress myeloma cell growth. Data from the APEX trial showed that grade 3 and 4 neutropenia occurred in $14 \%$ of patients. It was transient in most cases. G-CSF treatment was permitted and used in $6 \%$ of patients and incidence of febrile neutropenia was only $1 \%$. (49)

\section{Conclusions}

Severe neutropenia remains a very important problem for all specialties of medicine. In oncology, it is the common and dose limiting toxicity with chemotherapy because of the risk of severe bacterial and fungal infections. Prophylactic use of G-CSF can shorten the course of chemotherapy-induced neutropenia and prevent many of these infections. G-CSF is also an effective long-term therapy for several forms of chronic neutropenia. Antibiotics remain the mainstay for the treatment of infections in neutropenic patients, and their use for prophylaxis in "low risk" patients is growing.

\section{References}

1**. Crawford J, Dale DC, Kuderer NM, et al. Risk and timing of neutropenic events in adult cancer patients receiving chemotherapy: the results of a prospective nationwide study of oncology practice. J Natl Compr Canc Netw. 2008; 6:109-118. An important report emphasizing the risk of severe neutropenia in the first cycle of chemotherapy based on a large prospective study. [PubMed: 18319047]

$2 * *$. Lyman GH, Crawford J, Kuderer NM, et al. Final risk prediction model for neutropenic complications in patients receiving cancer chemotherapy. Blood. 2008; 112:474-5. (abstract). Preliminary report on the accuracy of a risk model for predicting the risk of severe neutropenia with cancer chemotherapy.

3. Pettengell R, Bosly A, Szucs TD, et al. Multivariate analysis of febrile neutropenia occurrence in patients with non-Hodgkin lymphoma: data from the INC-EU Prospective Observational European Neutropenia Society. Br J Haematol. 2009; 144:677-685. [PubMed: 19055662]

4. Dranitsaris G, Rayson D, Vincent M, et al. Identifying patients at high risk for neutropenic complications during chemotherapy for metastatic breast cancer with doxorubicin or pegylated liposomal doxorubicin: the development of a prediction model. Am J Clin Oncol. 2008; 31:369_ 374.12. [PubMed: 18845996] 
5*. Wicki S, Keisker A, Aebi C, et al. Risk prediction of fever in neutropenia in children with cancer: a step towards individually tailored supportive therapy? Pediatr Blood Cancer. 2008; 51:778-783. A pediatric study showing the specific factors associated with chemotherapy -induced neutropenia. [PubMed: 18726920]

6. Stabell N, Nordal E, Stensvold E, et al. Febrile neutropenia in children with cancer: a retrospective Norwegian multicentre study of clinical and microbiological outcome. Scand J Infect Dis. 2008; 40:301-307. [PubMed: 17918015]

7. Camps IR. Risk factors for invasive fungal infections in haematopoietic stem cell transplantation. Int J Antimicrob Agents. 2008; 32:S119-123. [PubMed: 19013335]

8**. Rüping MJ, Vehreschild JJ, Comely QA. Patients at high risk of invasive fungal infections: when and how to treat. Drugs. 2008; 68:1941-1962. An important report with recommendations for treatment of patients witrh suspected or proven fungal infections complicating chemotherapyinduced neutropenia, stem cell transplantation or leukemia. [PubMed: 18778118]

9. Nivolx Y, Velten M, Letscher-Bru V, et al. Factors associated with overall and attributable mortality in invasive aspergillosis. Clin Infect Dis. 2008; 47:1176-1184. [PubMed: 18808352]

10. Cheong HS, Kang CI, Wi YM, et al. Clinical significance and predictors of community-onset Pseudomonas aeroginosa bacteremia. Am J Med. 2008; 121:709-714. [PubMed: 18691485]

$11^{* *}$. Carstensen M, Sorensen JB. Outpatient management of febrile neutropenia: time to revise the present treatment strategy. J Support Oncol. 2008; 6:199-208. A good review of the uses of antibiotics and out patient management. [PubMed: 18551855]

12. Freifeld A, Sankaranarayanan J, Ullrich F, Sun J. Clinical practice patterns of managing low-risk adult febrile neutropenia during cancer chemotherapy in the USA. Support Care Cancer. 2008; 16:181-191. [PubMed: 17943327]

13. Pentheroudakis G, Fountzilas G, Kalofonos HP, et al. Palliative chemotherapy in elderly patients with common metastatic malignancies: A Hellenic Cooperative Oncology Group registry analysis of management, outcome and clinical benefit predictors. Crit Rev Oncol Hematol. 2008; 66:237247. [PubMed: 18243010]

14**. Lyman GH. Impact of chemotherapy dose intensity of cancer patient outcomes. J Natl Compre Cancer Netw. 2009; 7:99-108. An excellent review of the evidence and rationale for giving full, standard dose chemotherapy in the treatment of cancer.

15. Khan S, Dhadda A, Fyfe D, Sundar S. Impact of neutropenia on delivering planned chemotherapy for solid tumours. Eur J Cancer Care. 2008; 17:19-25.

$16^{* *}$. Wagner LI, Beaumont JL, Ding B, et al. Measuring health-related quality of life and neutropenia-specific concerns among older adults undergoing chemotherapy: validation of the functional Assessment of Cancer Therapy-Neutropenia (FACT-N). Support Care Cancer. 2008; 16:47-56. An important report describing the FACT-N assessment tool for evaluation the effects of neutropenia on patients' quality of life. [PubMed: 17619911]

17**. Greil R, Psenak O, Roila F. on behalf of the ESMO Guidelines Working Group. Hematopoietic growth factors: ESMO recommendations for the applications. Ann Oncol. 2008; 19:116-118. Important new guidelines.

$18^{* *}$. Kouroukis CT, Chia S, Verma S, et al. Canadian supportive care recommendations for the management of neutropenia in patients with cancer. Curr Oncol. 2008; 15:9-23. Useful new guidelines. [PubMed: 18317581]

19. Crawford J, Althaus B, Armitage J, et al. for the National Comprehensive Cancer Network (NCCN). Myeloid growth factors: Clinical practice guidelines in oncology. J Natl Compr Canc Netw. 200; 5:188-202. [PubMed: 17335688]

20. Smith TJ, Khatcheressian J, Lyman GH, et al. 2006 update of recommendations for the use of white blood cell growth factors: an evidence-based clinical practice guideline. J Clin Oncol. 2006; 24:3187-205. [PubMed: 16682719]

21. Bohlius J, Herbst C, Reiser M, et al. Granulopoiesis-stimulating factors to prevent adverse effects in the treatment of malignant lymphoma. Cochrane Database Syst Rev. 2008; 8:CD003189. [PubMed: 18843642] 
22. Kearney N, Friese C. Clinical practice guidelines for the use of colony-stimulating factors in cancer treatment: implications for oncology nurses. Eur J Oncol Nurs. 2008; 12:14-25. [PubMed: 18291720]

23*. Ramsey SD, Liu Z, Boer R, et al. Cost-effectiveness of primary versus secondary prophylaxis with pegfilgrastim in women with early-stage breast cancer receiving chemotherapy. Value Health. 2009; 12:217-225. Well-designed study of cost-effectiveness for supportive care with peg-G-CSF. [PubMed: 18673353]

24. Eldar-Lissal A, Cosler LE, Culakova E, Lyman GH. Economic analysis of prophylactic pegfilgrastim in adult cancer patients receiving chemotherapy. Value Health. 2008; 11:172-179. [PubMed: 18380630]

25. Timmer-Bonte JN, Adang EM, Termeer E, et al. Modeling the cost effectiveness of secondary febrile neutropenia prophylaxis during standard-dose chemotherapy. J Clin Oncol. 2008; 26:290296. [PubMed: 18182670]

26. Cosier LE, Eldar-Lissal A, Culakova E, et al. Therapeutic use of granulocyte colony-stimulating factors for established febrile neutropenia: effect on costs from a hospital perspective. Pharmacoeconomics. 2007; 25:343-351. [PubMed: 17402806]

27**. Lyman GH, Dale DC, Culakova E, et al. Acute myeloid leukemia or myelodysplastic syndrome (AML/MDS) and overall mortality with chemotherapy (CT) and granulocyte colony-stimulating factor (G-CSF): a metastatic-analysis of randomized controlled trials (RCTs). ASCO abstracts. 2009 (in press). An important meta-analysis of the risk of secondary leukemia associated with cancer treatments and supportive care.

28. Abernethy AP, Barbour SY, Uronis H, et al. Quality management of potential chemotherapyinduced neutropenia complications: evaluation of practice in an academic medical center. Support Care Cancer. 2008 Dec 19. Epub ahead of print.

29. Zuckerman J, Moreira LB, Stoll P, et al. Compliance with a critical pathway for the management of febrile neutropenia and impact on clinical outcomes. Ann Hematol. 2008; 87:139-145. [PubMed: 17938926]

30**. Andersohn F, Konzen C, Garbe E. Systematic review: agranulocytosis induced by nonchemotherapy drugs. Ann Intern Med. 2007; 146:657-665. An important and comprehensive review. [PubMed: 17470834]

31 * Andres E, Maloisel F. Idiosyncratic drug-induced agranulocytosis or acute neutropenia. Curr Opin Hematol. 2008; 15:15-21. An excellent recent summary of agranulocytosis from international experts. [PubMed: 18043241]

32. Antonini MG, Babudieri S, Maida I, et al. Incidence of neutropenia and infections during combination treatment of chronic hepatitis $C$ with pegylated interferon alfa- $2 a$ or alfa- $2 b$ plus ribavirin. Infection. 2008; 36:250-255. [PubMed: 18458815]

33. Babadoko AA, Aminu SM, Suleiman AN. Neutropenia and human immunodeficiency virus-1 infection: analysis of 43 cases. Niger J Med. 2008; 17:57-60. [PubMed: 18390135]

34*. Ortega M, Aimela M, Soriano A, et al. Bloodstream infections among human immunodeficiency virus-infected adult patients: epidemiology and risk factors for mortality. Eur J Clin Microbiol Infect Dis. 2008; 27:969-976. A useful report on current patterns of bacteremia in HIV patients in Spain. [PubMed: 18449581]

35*. Rosenberg PS, Alter BP, Link DC, et al. Neutrophil elastase mutations and risk of leukaemia in severe chronic neutropenia. Br J Haematol. 2008; 140:210-213. A report showing that the risk of leukemic evolution in severe congenital neutropenia is disease related and not specifically related to mutations of the ELA-2 gene. [PubMed: 18028488]

36**. Dale DC, Link DC. The many causes of severe congenital neutropenia. N Engl J Med. 2009; 360:3-5. A useful brief summary on congenital neutropenia, its mechanisms and treatment. [PubMed: 19118300]

37**. Zeidler C, Germeshausen M, Klein C, Welte K. Clinical implications of ELA2-, HAX1-, and GCSF-receptor (CSF3R) mutations in severe congenital neutropenia. Br J Haematol. 2009; 144:459-67. A useful brief summary on congenital neutropenia, its mechanisms and treatment. [PubMed: 19120359] 
38. Seidel MG, Peters C, Wacker A, et al. Randomized Phase III study of granulocyte transfusions in neutropenic patients. Bone Marrow Transplant. 2008; 42:679-84. [PubMed: 18695660]

39. Ofran Y, Avivi I, Oliven Q, et al. Granulocyte transfusions for neutropenic patients with lifethreatening infections: A single center experience in 47 patients, who received 348 transfusions. Vox Sang. 2007; 93:363-9. [PubMed: 18070282]

40*. Dale DC, Price TH. Granulocyte transfusion therapy: a new era? Curr Op Hematol. 2009; 16:1-2. A summary of the current status of granulocyte transfusions as treatment for neutropenia and announcement the currently on-going NIH sponsored randomized trial.

41. Price, Price TH.; Gurkamal, S.; Chatta, GS.; Dale, DC. The effect of recombinant granulocyte colony-stimulating factor on neutrophil kinetics in normal young and elderly humans. Blood. 1996; 88:335-340. [PubMed: 8704192]

42. Dale DC, Liles WC, Llewellyn C, Price TH. The effects of granulocyte-macrophage colonystimulating factor (GM-CSF) on neutrophil kinetics and function in normal human volunteers. Am J Hematol. 1998; 57:7-15. [PubMed: 9423810]

43. Smith TJ, Khatcheressian J, Lyman GH, et al. 2006 update of recommendations for the use of white blood cell growth factors: an evidence-based clinical practice guideline. J Clin Oncol. 2006; 24:3187-205. [PubMed: 16682719]

44**. Dale DC. Neutrophil biology and the next generation of myeloid growth factors. J Natl Compr Cane Netw. 2009; 7:92-98. A review of efforts to develop new versions of G-CSF.

45. Roger SD, Goldsmith D. Biosimilars: it's not as simple as cost alone. J Clin Pharm Ther. 2008; 33:459-464. [PubMed: 18834359]

46. Lai GG, Lim ST, Tao M, et al. Late-onset neutropenia following RCHOP chemotherapy in diffuse large B-cell lymphoma. Am J Hematol. 2009 Mar 31. Epub ahead of print.

47**. Ocio EM, Mateos MV, Maiso P, et al. New drugs in multiple myeloma: mechanisms of action and phase I/II clinical findings. Lancet Oncol. 2008; 12:1157-65. Excellent review article. [PubMed: 19038762]

48. Wang M, Dimopoulos MA, Chen C, et al. Lenalidomide plus dexamethasone is more effective than dexamethasone alone in patients with relapsed or refractory multiple myeloma regardless of prior thalidomide exposure. Blood. 2008; 112:4445-51. [PubMed: 18799726]

49. Lonial S, Richardson PG, San Miguel J, et al. Characterisation of haematological profiles and low risk of thromboembolic events with bortezomib in patients with relapsed multiple myeloma. $\mathrm{Br} \mathrm{J}$ Haematol. 2008; 143:222-9. [PubMed: 18713253] 